\title{
Lower LDL-cholesterol levels associated with increased inflammatory burden in patients with acute ST-segment elevation myocardial infarction
}

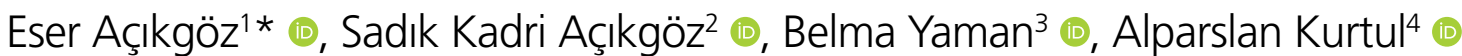

\begin{abstract}
SUMMARY
OBJECTIVE: Association of low-density lipoprotein cholesterol and highly sensitive C-reactive protein in ST-elevation myocardial infarction patients was assessed in this study.

METHODS: 591 consecutive patients who were hospitalized with a diagnosis of ST-elevation myocardial infarction were enrolled and assigned into tertiles according to their serum low-density lipoprotein cholesterol levels. Differences in highly sensitive C-reactive protein among low-density lipoprotein cholesterol tertiles and correlations between highly sensitive C-reactive protein and low-density lipoprotein cholesterol were assessed.

RESULTS: Highly sensitive $C$-reactive protein levels differed significantly among the groups $(p<0.001)$ and found to be highest in the lowdensity lipoprotein cholesterol tertile 1 and lowest in the low-density lipoprotein cholesterol tertile 3 (post-hoc p-values: tertile 1 vs. 2 $<0.001$; tertile 1 vs. $3<0.001$; tertile 2 vs. $3=0.019$ ). There was a negative correlation between $h s-C R P$ and both low-density lipoprotein cholesterol $(r=-0.332, p<0.001)$ and total cholesterol $(r=-0.326, p<0.001)$. There was also a negative correlation between highly sensitive $C$-reactive protein and high-density lipoprotein cholesterol, though the strength of this relationship was weak $(r=-0.103, p=0.014)$.

CONCLUSION: Lower low-density lipoprotein cholesterol levels are associated with higher inflammatory burden in patients with acute STEMI. Further studies are required to elucidate the significance of low-density lipoprotein cholesterol levels in ST-elevation myocardial infarction settings.

KEYWORDS: ST elevation myocardial infarction. Cholesterol, LDL. Hs-CRP.
\end{abstract}

\section{INTRODUCTION}

Elevated low-density lipoprotein cholesterol (LDL) is one of the most emphasized risk factors for cardiovascular disease (CVD). Results of the studies evaluating the effect of statins on LDL reduction led up to the motto "the lower the better" for prevention of $\mathrm{CVD}^{1,2}$. Inversely, lower LDL levels were noticed in acute myocardial infarction (AMI) patients for more than 50 years $^{3,4}$. Subsequent studies revealed that serum cholesterol levels fall rapidly after an AMI such that serum LDL decreased $48 \%$ below baseline on the $7^{\text {th }}$ day of $\mathrm{AMI}^{5-8}$. Moreover, several studies showed worse clinical outcomes in AMI and heart failure patients with lower total cholesterol (TC) and LDL levels indicated a "cholesterol paradox" ${ }^{\text {"14. }}$. Previously, several clinical studies demonstrated

\footnotetext{
'Dr. Abdurrahman Yurtaslan Ankara Oncology Training and Research Hospital, Department of Cardiology - Ankara, Turkey.

${ }^{2}$ Yıldırım Beyazıt University Yenimahalle Education and Research Hospital, Department of Cardiology - Ankara, Turkey.

${ }^{3}$ Near East University, Faculty of Medicine, Department of Cardiology - Lefkosa, Turkey.

${ }^{4}$ Hatay Mustafa Kemal University, Department of Cardiology - Hatay, Turkey.

*Corresponding author: dreacikgoz@gmail.com

Conflicts of interest: the authors declare there are no conflicts of interest. Funding: none.

Received on October 07, 2020. Accepted on December 10, 2020.
} 
the acute phase reactant properties of serum lipoproteins in bacterial and viral infections in humans ${ }^{15,16}$. Alterations in LDL levels in heart failure and its prognostic impact were also largely explained by acute phase reactant properties of serum lipoproteins ${ }^{10}$. Thus, LDL levels may have the potential to be an inflammatory marker in AMI. However, association of LDL and inflammatory burden in AMI patients were not assessed before. Thus, the relationship of serum LDL with highly sensitive C-reactive protein (hs-CRP) in patients with ST-segment elevation myocardial infarction (STEMI) was investigated.

\section{METHODS}

We retrospectively enrolled 591 consecutive patients who were hospitalized with a diagnosis of STEMI. Patients were enrolled into the study if their hs-CRP levels and fasting lipid profiles were available within 24 hours after the onset of symptoms. Medical history of the patients were obtained from medical records. Forty six patients were excluded from the study due to the use of lipid-lowering medications. In order to perform analysis, the patients were assigned into tertiles according to their serum LDL levels. STEMI was defined as $\geq 1 \mathrm{~mm}$ ST-segment elevation in at least two contiguous electrocardiogram leads, except V2-V3, which required $1.5 \mathrm{~mm}$ for female patients, $2 \mathrm{~mm}$ for male patients $>40$ years of age, and $2.5 \mathrm{~mm}$ for male patients $<40$ years old or new onset left bundle-branch block in the presence of ischemia symptoms. A transthoracic echocardiography was performed 48 to 72 hours after admission using a Vivid 7 system (GE Medical Systems, Milwaukee, WI, USA) and the left ventricular ejection fraction was calculated by the modified Simpson method. Serum levels of hs-CRP were measured using the latex enhanced immune-turbidimetric method (Cardio-Phase High Sensitivity C-Reactive Protein; Siemens Healthcare Diagnostics Inc., Tarrytown, New York, USA). Serum TC, triglyceride (TG), and high-density lipoprotein cholesterol (HDL-C) concentrations were analyzed by a BM-Hitachi-747 auto-analyzer (Boehringer Mannheim $\mathrm{GmbH}$, Mannheim, Germany). Serum LDL-C values were estimated by the formula of Friedewald et al. or directly measured if TG $>400 \mathrm{mg} / \mathrm{dL}^{17}$. The study protocol was approved by the Local Ethics Committee.

SPSS Statistics, version 17.0 (SPSS Inc, Chicago, IL), was used for statistical analysis. Kolmogorov-Smirnov test was used to determine the distribution patterns. Data were presented as mean and standard deviation, median and interquartile range, or proportions as appropriate. The one-way ANOVA was used to compare data with normal distribution and Kruskal-Wallis test was used to compare the data without normal distribution. Bonferroni correction was used for multiple comparisons. Pearson's correlation analysis was used to assess the correlation between hs-CRP and serum lipid parameters. Categorical variables were compared with the $\chi^{2}$ test. A two-tailed $p$-value $<0.05$ was considered to be statistically significant.

\section{RESULTS}

Every LDL tertile contains 197 patients. Mean age of the participants was 61.71. There was no difference in regard to demographic characteristics among the LDL tertiles. Time to lipid measurement was also similar. Mean LDL level was $75.59 \pm 17.207$ for tertile $1,115.79 \pm 9.487$ for tertile 2 , and $161.61 \pm 25.968$ for tertile 3 (Table 1). hs-CRP levels differed significantly among the groups $(\mathrm{p}<0.001)$ and found to be highest in tertile 1 and lowest in tertile 3 (post-hoc p-values: tertile 1 vs. $2<0.001$; tertile 1 vs. $3<0.001$; tertile 2 vs. $3=0.019$ ). Mean troponin T level was $25.3 \pm 14.7$ in tertile 1 , $19.5 \pm 15.5$ in tertile 2 , and $16.6 \pm 12.2$ in tertile $3(\mathrm{p}=0.01$ ). There was a negative correlation between hs-CRP and both LDL $(\mathrm{r}=-0.332, \mathrm{p}<0.001)$ and TC $(\mathrm{r}=-0.326, \mathrm{p}<0.001)$. There was also a negative correlation between hs-CRP and HDL, though the strength of this relationship was weaker $(\mathrm{r}=-0.103, \mathrm{p}=0.014)$. There was no correlation between hs-CRP and TG. (Figure 1)

\section{DISCUSSION}

In the present study, the association of serum LDL with hs-CRP in STEMI patients was association and it was found that hs-CRP levels are higher in the lowest LDL tertile and lower in the highest LDL tertile. In addition, there was a negative correlation between LDL and hs-CRP in this patient group. Moreover, hs-CRP was negatively correlated with both TC and HDL. Troponin T levels were also higher in the lowest LDL tertile and lower in the highest LDL tertile. To the best of our knowledge, this the first study in the literature revealing the inverse association of hs-CRP with serum LDL in STEMI patients.

Previous studies showed that marked changes in serum lipoproteins occur during the course of AMI including reductions in TC, $\mathrm{LDL}$, and $\mathrm{HDL}$ and increases in $\mathrm{TG}^{4-8}$. Infarction size is probably important in reducing LDL, as shown in the study of Rott et al., which suggested that LDL levels decrease significantly after an AMI and the reduction is correlated with cardiac troponin $T$ levels ${ }^{18}$. Several mechanisms were proposed to explain the lipoprotein alterations after AMI. First, it was shown that acute phase response causes up-regulation of $\mathrm{LDL}$ receptor 
Table 1. Demographic, clinical, and laboratory characteristics of study participants.

\begin{tabular}{|c|c|c|c|c|}
\hline & Tertile $1, \mathrm{n}=197$ & Tertile 2, n=197 & Tertile 3, n=197 & p-value \\
\hline Age (years) & $62.93 \pm 11.62$ & $61.79 \pm 13.05$ & $60.43 \pm 11.39$ & 0.09 \\
\hline Male gender - n (\%) & $154(78.2)$ & $136(69.90)$ & $133(67.5)$ & 0.055 \\
\hline Body mass index $\left(\mathrm{kg} / \mathrm{m}^{2}\right)$ & $28.0 \pm 4.6$ & $28.0 \pm 4.3$ & $28.0 \pm 4.4$ & 0.319 \\
\hline Hypertension - n (\%) & $80(40.6)$ & $88(44.7)$ & $83(42.1)$ & 0.712 \\
\hline Diabetes - n (\%) & $77(39.1)$ & $62(31.5)$ & 62 (31.5) & 0.183 \\
\hline Smoking - n (\%) & $71(36.0)$ & $93(47.2)$ & $100(50.8)$ & 0.009 \\
\hline Prior $\mathrm{Ml}-\mathrm{n}(\%)$ & $19(9.6)$ & $8(4.1)$ & $14(7.1)$ & 0.092 \\
\hline Prior stroke - n (\%) & $8(4.1)$ & $5(2.5)$ & $6(3.0)$ & 0.600 \\
\hline Glucose (mg/dL) & $160.5 \pm 83.9$ & $153.6 \pm 81.5$ & $143.6 \pm 72.5$ & 0.107 \\
\hline Creatinine $(\mathrm{mg} / \mathrm{dL})$ & $1.12 \pm 26$ & $1.09 \pm 25$ & $1.03 \pm 21$ & 0.001 \\
\hline CK-MB (mg/dL) & $75.14 \pm 39.76$ & $77.88 \pm 39.41$ & $76.71 \pm 39.11$ & 0.955 \\
\hline Troponin T & $25.3 \pm 14.7$ & $19.5 \pm 15.5$ & $16.6 \pm 12.2$ & 0.010 \\
\hline Cholesterol (mg/dL) & $143.0 \pm 23.6$ & $187.8 \pm 17.8$ & $241.8 \pm 41.9$ & $<0.001$ \\
\hline LDL-C (mg/dL) & $75.59 \pm 17.2$ & $115.79 \pm 9.5$ & $161.61 \pm 25.9$ & $<0.001$ \\
\hline $\mathrm{HDL}-\mathrm{C}(\mathrm{mg} / \mathrm{dL})$ & $38.33 \pm 9.922$ & $41.45 \pm 9.312$ & $42.84 \pm 9.613$ & $<0.001$ \\
\hline Triglyceride (mg/dL) & $142.1 \pm 78.3$ & $152.6 \pm 76.6$ & $185.7 \pm 110.4$ & $<0.001$ \\
\hline $\mathrm{Hs}-\mathrm{CRP}$ (mg/L) & $7.19 \pm 3.38$ & $5.93 \pm 2.98$ & $5.09 \pm 2.76$ & $<0.001$ \\
\hline Hemoglobin (g/dL) & $14.16 \pm 1.53$ & $14.43 \pm 1.46$ & $14.54 \pm 1.44$ & 0.061 \\
\hline WBC $(x 10,000 / \mathrm{mL})$ & $10.89 \pm 3.50$ & $10.85 \pm 3.42$ & $10.59 \pm 3.33$ & 0.639 \\
\hline Platelets $(x 1,000 / \mathrm{mL})$ & $222.29 \pm 65.48$ & $240.46 \pm 62.38$ & $243.05 \pm 59.47$ & 0.002 \\
\hline LVEF (\%) & $46.20 \pm 11.78$ & $48.44 \pm 9.44$ & $48.26 \pm 9.93$ & 0.080 \\
\hline Pulse rate $\left(\mathrm{min}^{-1}\right)$ & $79.93 \pm 15.54$ & $79.89 \pm 14.83$ & $79.16 \pm 13.56$ & 0.842 \\
\hline Systolic BP (mmHg) & $124.97 \pm 22.01$ & $131.39 \pm 24.03$ & $132.32 \pm 26.94$ & 0.005 \\
\hline Diastolic BP $(\mathrm{mmHg})$ & $76.43 \pm 13.073$ & $79.17 \pm 13.425$ & $79.71 \pm 15.062$ & 0.044 \\
\hline Syntax Score & $16.69 \pm 10.51$ & $15.36 \pm 9.25$ & $15.08 \pm 9.01$ & 0.210 \\
\hline Time to lipid measurement (h) & $6(4-8)$ & $7(4-8)$ & $6(4-8)$ & 0.374 \\
\hline \multicolumn{5}{|l|}{ Initial Treatment } \\
\hline Medical-Fibrinolytic & $6(3.0)$ & $4(2.0)$ & $9(4.5)$ & \multirow{3}{*}{0.356} \\
\hline $\mathrm{PCl}$-stent & $162(82.2)$ & $172(87.3)$ & $168(85.3)$ & \\
\hline CABG & $29(14.8)$ & $21(10.7)$ & $20(10.2)$ & \\
\hline
\end{tabular}

MI: myocardial infarction; CK-MB: creatinine kinase myocardial band; LDL-C: low density lipoprotein cholesterol; HDL-C: high density lipoprotein cholesterol; hs-CRP: high sensitivity C-reactive protein; WBC: white blood cell; LVEF: left ventricular ejection fraction; BP: blood pressure; PCI: percutaneous coronary intervention; CABG: coronary artery by-pass graft operation.

activity and reduction in some HDL regulatory proteins ${ }^{19,20}$. In addition, myocardial necrosis facilitates adrenergic-mediated adipocyte lipolysis leading to free fatty acid mobilization, increased hepatic very low density lipoprotein (VLDL) secretion, TG elevation, and alteration in LDL and HDL particle composition $^{21,22}$. Other possible contributors to lipid changes after AMI include in-hospital therapy and lifestyle changes such as heparin, which causes lipoprotein lipase-mediated TG hydrolysis, beta-blockers which suppress hormone-sensitive lipase, postural effects and reduction of saturated fat intake ${ }^{23-28}$. 

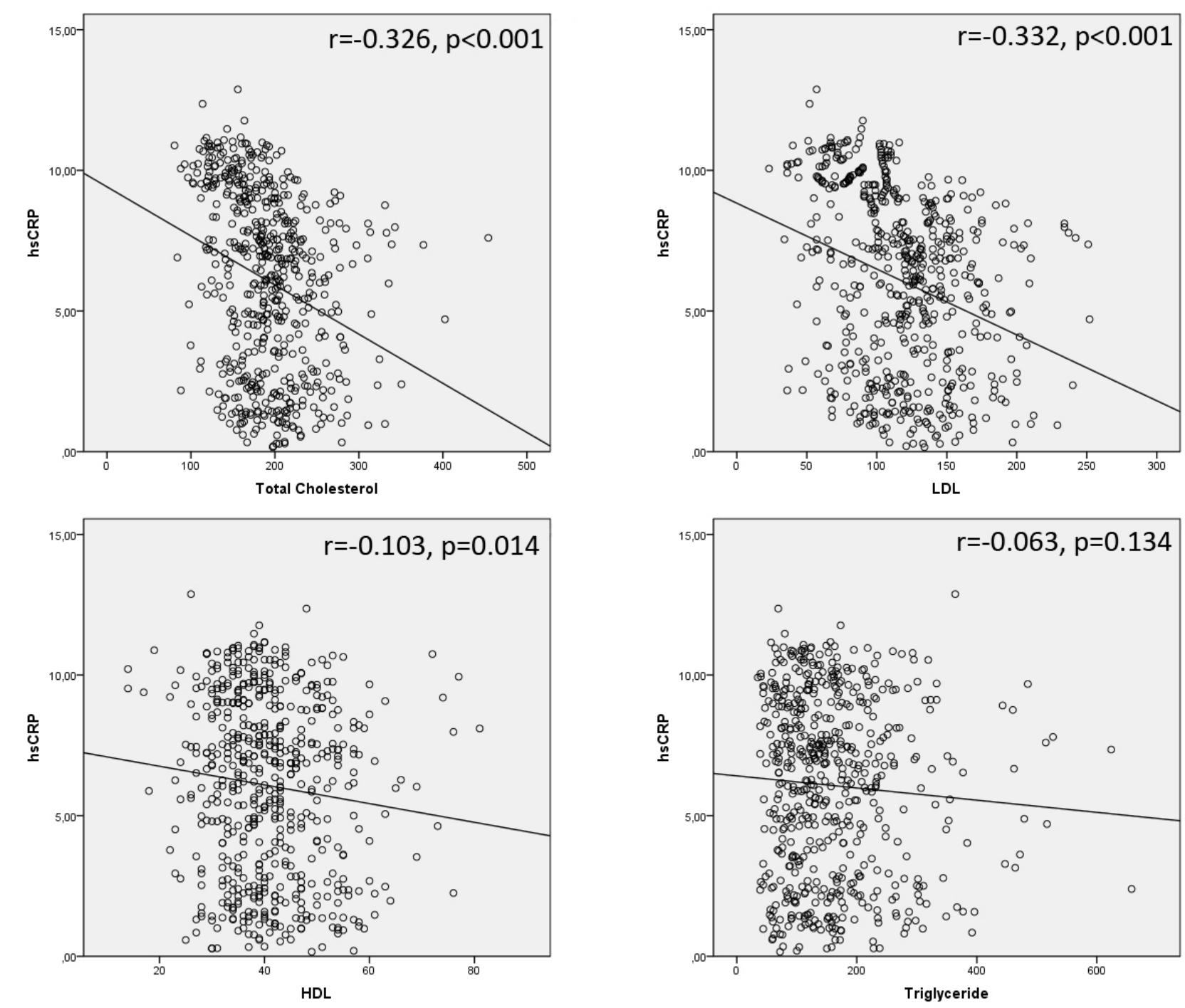

Figure 1. Scatterplots summarizing the correlations between hs-CRP and serum lipoproteins.

Impact of the low cholesterol levels on the prognosis of patients with CVD was investigated in some studies previously. In one of them, Richartz et al. found out that lower cholesterol levels were associated with poor survival in patients with advanced heart failure on mechanical support ${ }^{9}$. In another, Rauchhaus et al. demonstrated that lower cholesterol levels are associated with poor clinical outcome in patients with chronic heart failure regardless of the heart failure etiology. In addition, negative correlation of serum cholesterol and tumor necrosis factor alpha was revealed in this study ${ }^{10}$. A similar result was found in the study by Norwich et al., which showed that low serum TC, LDL, HDL, and TG are related to increased mortality in advanced heart failure ${ }^{11}$. In two studies about low LDL levels in myocardial infarction, Al-Mallah et al. found out that low LDL levels are associated with increased 3-year mortality in non STEMI and Reddy at al. demonstrated that low LDL levels are associated with higher in-hospital mortality after AMI ${ }^{12-14}$.

Results of the present study revealing the relationship of hs-CRP and lipoprotein levels, support the hypothesis that AMI patients with lower LDL have higher inflammatory burden and acute phase response is responsible for lipoprotein changes after AMI. Cho et al. demonstrated that life-saving medications, including lipid-lowering drugs, were underused in MI patients with lower admission LDL levels ${ }^{13}$. Considering these patients have higher hs-CRP levels, hesitation to initiate adequate doses of lipid-lowering therapy due to low LDL levels may contribute to a worse prognosis. 
The present study has several limitations that should be taken into account when interpreting its results. First, it is a retrospective and single center study. However, all consecutive cases in a period of time were enrolled in order to eliminate selection bias. In addition, preadmission serum lipoprotein levels of the patients were not available and serial measurements of LDL were not performed. Thus, exact changes in serum lipoproteins due to AMI were not available.

\section{CONCLUSIONS}

Results of the present study suggest that acute STEMI patients with lower LDL levels have higher inflammatory burden reflected by higher hs-CRP levels. Further prospective studies are needed to confirm this hypothesis and elucidate the clinical significance of the findings of this study.

\section{AUTHORS" CONTRIBUTIONS}

EA: Conceptualization, Data Curation, Formal Analysis, Writing - Original Draft, Writing - Review \& Editing. SKA: Conceptualization, Data Curation, Formal Analysis, Writing - Original Draft, Writing - Review \& Editing. BY: Conceptualization, Data Curation, Writing - Original Draft, Writing - Review \& Editing. AK: Conceptualization, Formal Analysis, Writing - Original Draft, Writing - Review \& Editing.

\section{REFERENCES}

1. Expert panel on detection, evaluation, and treatment of high blood cholesterol in adults. Executive summary of the third report of the national cholesterol education program (NCEP) expert panel on detection, evaluation, and treatment of high blood cholesterol in adults (adult treatment panel III). JAMA. 2001;285(19):2486-97. https://doi.org/10.1001/ jama.285.19.2486

2. Grundy SM, Stone NJ, Bailey AL, Beam C, Birtcher KK, Blumenthal RS, et al. 2018 AHA/ACC/AACVPR/AAPA/ABC/ ACPM/ADA/AGS/APhA/ASPC/NLA/PCNA guideline on the management of blood cholesterol: a report of the American College of Cardiology/American Heart Association Task Force on clinical practice guidelines. Circulation. 2019;139(25):e1082143. https://doi.org/10.1161/CIR.0000000000000625

3. Biorck G, Blomqvist G, Sievers J. Cholesterol values in patients with myocardial infarction and in a normal control group. Acta Med Scand. 1957;156(6):493-7. https://doi. org/10.1111/j.0954-6820.1957.tb00107.x

4. Dodds C, Mills GL. Influence of myocardial infarction on plasmalipoprotein concentration. Lancet. 1959;1(7084):1160-3. https://doi.org/10.1016/s0140-6736(59)91178-x

5. Watson WC, Buchanan KD, Dıckson C. serum cholesterol levels after myocardıal ınfarctıon. Br Med J. 1963;2(5359):709-12. https://doi.org/10.1136/bmj.2.5359.709

6. Fyfe T, Baxter RH, Cochran KM, Booth EM. Plasma-lipid changes after myocardial infarction. Lancet. 1971;2(7732):997-1001. https://doi.org/10.1016/s0140-6736(71)90322-9

7. Heldenberg D, Rubinstein A, Levtov O, Berns L, Werbin B, Tamir I. Serum lipids and lipoprotein concentrations during the acute phase of myocardial infarction. Atherosclerosis. 1980;35(4):433-7. https://doi.org/10.1016/00219150(80)90184-7

8. Rosenson RS. Myocardial injury: the acute phase response and lipoprotein metabolism. J Am Coll Cardiol. 1993;22(3):933-40. https://doi.org/10.1016/0735-1097(93)90213-k

9. Richartz BM, Radovancevic B, Frazier $\mathrm{OH}$, Vaughn WK, Taegtmeyer H. Low serum cholesterol levels predict high perioperative mortality in patients supported by a left-ventricular assist system. Cardiology. 1998;89(3):184-8. https://doi. org/10.1159/000006785

10. Rauchhaus M, Koloczek V, Volk H, Kemp M, Niebauer J, Francis DP, et al. Inflammatory cytokines and the possible immunological role for lipoproteins in chronic heart failure. Int J Cardiol. 2000;76(2-3):125-33. https://doi.org/10.1016/ s0167-5273(00)00224-2

11. Horwich TB, Hamilton MA, Maclellan WR, Fonarow GC. Low serum total cholesterol is associated with marked increase in mortality in advanced heart failure. J Card Fail. 2002;8(4):21624. https://doi.org/10.1054/jcaf.2002.0804216

12. Al-Mallah MH, Hatahet $\mathrm{H}$, Cavalcante JL, Khanal S. Low admission LDL-cholesterol is associated with increased 3-year all-cause mortality in patients with non ST segment elevation myocardial infarction. Cardiol J. 2009;16(3):227-33. PMID: 19437396

13 Cho KH, Jeong MH, Ahn Y, Kim YJ, Chae SC, Hong TJ, et al. Korea Acute Myocardial Infarction Registry Investigators. Low-density lipoprotein cholesterol level in patients with acute myocardial infarction having percutaneous coronary intervention (the cholesterol paradox). Am J Cardiol. 2010;106(8):1061-8. https://doi.org/10.1016/j.amjcard.2010.06.009

14. Reddy VS, Bui QT, Jacobs JR, Begelman SM, Miller DP, French WJ. Relationship between serum low-density lipoprotein cholesterol and in-hospital mortality following acute myocardial infarction (the lipid paradox). Am J Cardiol. 2015;115(5):55762. https://doi.org/10.1016/j.amjcard.2014.12.006

15. Tanaka S, Diallo D, Delbosc S, Genève C, Zappella N, YongSang J, et al. High-density lipoprotein (HDL) particle size and concentration changes in septic shock patients. Ann Intensive Care. 2019;9(1):68. https://doi.org/10.1186/s13613-0190541-8

16. Lima WG, Souza NA, Fernandes SOA, Cardoso VN, Godói IP. Serum lipid profile as a predictor of dengue severity: a systematic review and meta-analysis. Rev Med Virol. 2019;29(5):e2056. https://doi.org/10.1002/rmv.2056 
17. Friedewald WT, Levy RI, Fredrickson DS. Estimation of the concentration of low-density lipoprotein cholesterol in plasma, without use of the preparative ultracentrifuge. Clin Chem. 1972;18(6):499-502. PMID: 4337382

18. Balci $B$. The modification of serum lipids after acute coronary syndrome and importance in clinical practice. Curr Cardiol Rev. 2011;7(4):272-6. https://doi. org/10.2174/157340311799960690

19. Wattanasuwan N, Khan IA, Gowda RM, Vasavada BC, Sacchi TJ. Effect of acute myocardial infarction on cholesterol ratios. Chest. 2001;120(4):1196-9. https://doi.org/10.1378/ chest.120.4.1196

20. Rott D, Klempfner R, Goldenberg I, Leibowitz D. Cholesterol levels decrease soon after acute myocardial Infarction. Isr Med Assoc J. 2015;17(6):370-3. PMID: 26233997

21. Pfohl M, Schreiber I, Liebich HM, Häring HU, Hoffmeister HM. Upregulation of cholesterol synthesis after acute myocardial infarction - is cholesterol a positive acute phase reactant? Atherosclerosis. 1999;142(2):389-93. https://doi.org/10.1016/ s0021-9150(98)00242-1

22. Van Lenten BJ, Reddy ST, Navab M, Fogelman AM. Understanding changes in high density lipoproteins during the acute phase response. Arterioscler Thromb Vasc Biol. 2006;26(8):1687-8. https://doi.org/10.1161/01.ATV.0000232522.47018.a6
23. Large V, Peroni O, Letexier D, Ray H, Beylot M. Metabolism of lipids in human white adipocyte. Diabetes Metab. 2004;30(4):294309. https://doi.org/10.1016/s1262-3636(07)70121-0

24. Austin MA, King MC, Vranizan KM, Krauss RM. Atherogenic lipoprotein phenotype. A proposed genetic marker for coronary heart disease risk. Circulation. 1990;82(2):495-506. https:// doi.org/10.1161/01.cir.82.2.495

25. Mulder $M$, Lombardi $P$, Jansen $H$, van Berkel TJ, Frants RR, Havekes LM. Low density lipoprotein receptor internalizes low density and very low density lipoproteins that are bound to heparan sulfate proteoglycans via lipoprotein lipase. J Biol Chem. 1993;268(13):9369-75. PMID: 8387492

26. Day JL, Metcalfe J, Simpson CN. Adrenergic mechanisms in control of plasma lipid concentrations. Br Med J (Clin Res Ed). 1982;284(6323):1145-8. https://doi.org/10.1136/ bmj.284.6323.1145

27. Miller M, Bachorik PS, Cloey TA. Normal variation of plasma lipoproteins: postural effects on plasma concentrations of lipids, lipoproteins, and apolipoproteins. Clin Chem. 1992;38(4):56974. PMID: 1533184

28. Sacks FM, Katan M. Randomized clinical trials on the effects of dietary fat and carbohydrate on plasma lipoproteins and cardiovascular disease. Am J Med. 2002;113(Suppl 9B):13S-24S. https://doi.org/10.1016/s0002-9343(01)00987-1 